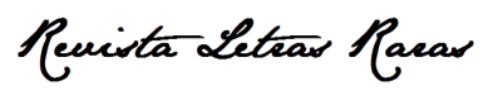

ISSN: 2317-2347 - v. 10, n. Spécial (2021)

Todo o conteúdo da RLR está licenciado sob Creative Commons Atribuição 4.0 Internacional

\title{
50 ans de la FIPF : la vie associative en réseau
}

\section{Denise Gisele de Britto Damasco*}

Licenciée en Lettres (1986), ayant un Master Recherche (2008) et Doctorat (2014) en Éducation à l'Université de Brasilia. Enseignante de français langue étrangère au Secrétariat d'État d'Éducation du DF de 1989 à 2015. Actuellement, suit des études postdoctorales à la PUC SP et préside la Fédération brésilienne des professeurs de français (2017-2020 et 2020/2022).

(iD) http://orcid.org/0000-0002-0250-0776

\section{Dario Pagel*}

Diplômé en Lettres portugais-français par la Fundação Universidade Regional de Blumenau (1974), master (1978), doctorat (1981), postdoctorat (1994) en Lettres - Phonétique générale et expérimentale par l'Université des Sciences Humaines de Strasbourg et Post- Docteur en Didactique des Langues et Cultures par l'Université de Paris III, Sorbonne Nouvelle (2004). II est actuellement professeur associé de langue et phonétique françaises et Directeur du Département des Lettres Etrangères de l'Université fédérale de Sergipe (UFS).

\section{Doina Spita**}

Enseignant-chercheur à I'Université "Al.I.Cuza" lasi (Roumanie) et Vice-présidente de la Fédération International des Professeurs de Français (FIPF), Doina Spiţa est spécialisée dans les sciences du langage, la didactique du français langue étrangère et l'intercompréhension. Elle participe à de nombreux projets de recherche en didactique du plurilinguisme et anime des formations de formateurs et des ateliers de sensibilisation à l'intercompréhension dans des espaces linguistiques et géographiques divers. Elle est auteur de nombreuses publications dans les domaines mentionnés ci-dessus.

\section{Waldemar Oliveira de Andrade Junior $r^{* * * t}$}

Master en Didactique de Langues et Cultures - Français langue étrangère et seconde, Université de ClermontAuvergne. Président de l'Association des Professeus de Français du District fédéral (2019/2021).

(iD) https://orcid.org/0000-0002-1130-8663

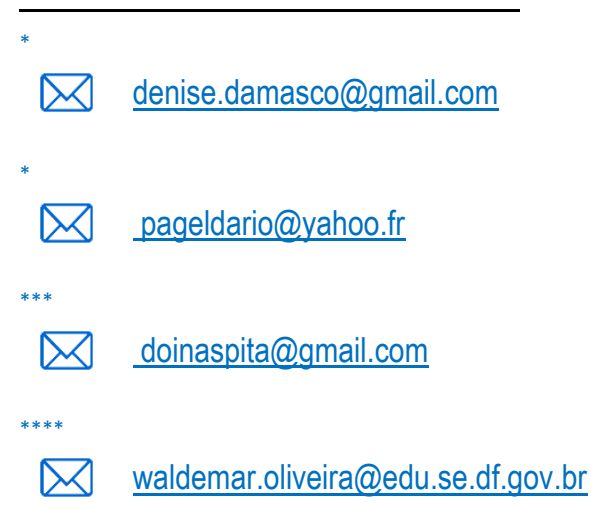




\section{Remita Letear Pacar}

ISSN: 2317-2347 - v. 10, n. Spécial (2021)

Todo o conteúdo da RLR está licenciado sob Creative Commons Atribuição 4.0 Internacional

Reçu en: 14 novembre 2021. Approuvé en: 16 novembre 2021.

\section{Comment citer cet article:}

DAMASCO, Denise Gisele de Britto. PAGEL, Dario. SPITA, Doina. JUNIOR, Waldemar Oliveira de Andrade. 50 ans de la FIPF : la vie associative en réseau. Revista Letras Raras. Campina Grande, v. 10, n. Spécial, p. 165183, nov. 2021.

\section{RÉSUMÉ}

Ce texte a pour but de réunir les témoignages qui nous permettent d'observer clairement que la vie associative se maintient et évolue dans différentes régions, même si l'on observe un recul du français dans le réseau scolaire. Les associations sont devenues une structure indispensable pour le développement de l'enseignement de cette langue étrangère. Dans tous les pays, nous pourrons trouver des exemples de leur travail politique et pédagogique fructueux que nous allons décrire lors de cet article. Cet article tient à souligner que les professeurs, par le biais du réseau des associations membres de la FIPF (Fédération Internationale des Professeurs de Français), mènent une action capitale en ce qui concerne l'enseignement et la formation des professeurs, donnant ainsi au français sa juste place dans l'enseignement plurilingue.

MOTS-CLÉS : Vie associative ; Formation des professeurs ; FLE

\section{RESUMO}

Este texto pretende reunir testemunhos que nos permitem observar claramente que a vida associativa se mantém e evolui nas diferentes regiões, ainda que observemos um recuo do francês na rede escolar. As associações tornaram-se uma estrutura essencial para o desenvolvimento do ensino desta língua estrangeira. Em todos os países, podemos encontrar exemplos de seu trabalho político e educacional de sucesso que descreveremos neste artigo. Este artigo busca a enfatizar que os professores, através da rede de associações membros da FIPF (Federação Internacional de Professores de Francês), realizam uma grande ação no que se refere ao ensino e à formação de professores, dando ao francês o seu devido lugar no ensino.

PALAVRAS-CHAVE: Vida associativa; Formação de professores; FLE

\section{Introduction}

Cet article rapporte la table-ronde qui a eu lieu pendant la 22ème édition du Congrès brésilien des professeurs de français, en 2019, à Brasilia, du 8 au 11 octobre. Nous vous proposons non seulement un bilan de ce qui a été présenté à l'occasion de ce Congrès, mais aussi un témoignage de trois professeurs et de leurs expériences significatives concernant la Fédération internationale des professeurs de français. Et un rapport d'expérience d'un jeune président qui était à l'audience à l'occasion de cette table-ronde. Celui-ci nous fait part de ses perceptions de la vie associative nous faisant parcourir cette passerelle entre les actions d'une association jusqu'aux actions de la Fédération internationale.

Selon Franchon (2019), ce Congrès, intitulé Le français en action : variation et créations, « de nature polyphonique, aura l'ambition d'aborder la langue française et son positionnement dans un 


\section{Remita Letear Pacar}

ISSN: 2317-2347 - v. 10, n. Spécial (2021)

Todo o conteúdo da RLR está licenciado sob Creative Commons Atribuição 4.0 Internacional

monde de mobilités constantes qui induisent de nouvelles dynamiques et de nouvelles stratégies » (FRANCHON, 2019, p. 147). Une série de conférences, de symposiums, de communications thématiques, de table-rondes et d'ateliers ont rythmé cette manifestation scientifique autours de cinq axes : la linguistique ; la formation et la didactique de langue ; la littérature et les passerelles culturelles ; la traduction et les politiques linguistiques ainsi que les mémoires partagées. Afin de fêter le cinquantième anniversaire de la FIPF, une table-ronde a eu lieu, ayant la participation de Patrick Dahlet, de Doina Spita et de Dario Pagel, sous la modération de Denise Damasco.

II faut souligner que les Actes de cet événement ont été lancés en 2020, sans que cette thématique ait été abordée, ce qui nous a motivés à mener cet effort pour rapporter ce qui s'est déroulé pendant le congrès et un témoignage individuel sur la FIPF et les parcours professionnels. II s'agit donc de soulever la vie associative en réseau, puisque la FIPF fédère des associations et des fédérations de professeurs de français du monde entier.

Cet article est conçu en deux parties. La première partie nous présente la FIPF et le réseau fédératif international. La seconde partie, nous invite à lire cinq témoignages sur la FIPF et ses 50 ans : celui du chercheur et son ancien président, Dario Pagel, de la présidente d'une Fédération adhérente à la FIPF, Denise Damasco, d'une association affiliée à la Fédération brésilienne, Waldemar Andrade de Oliveira Junior et par le témoignage de Doina Spita, vice-présidente de cette institution à l'époque. Soulignons que Mme Spita a été reconduite dans son poste de vice-présidente pour les années à venir : 2021 à 2025.

\section{Témoignages, vécus et expériences}

Nous vous présentons quatre témoignages sur la FIPF. Tout d'abord, Dario Pagel attire l'attention sur cette fédération et son rapport à la Francophonie. Ensuite, Denise Damasco nous dévoile ses perceptions en tant qu'enseignante et membre de bureau d'association adhérant à la FIPF, la Fédération brésilienne des professeurs de français (FBPF) et finalement le récit d'un président d'association affiliée à la FBPF et par conséquent à la FIPF. Nous y retrouverons aussi les vécus et expériences de la vice-présidente de la FIPF, Doina Spita. 


\section{Reuista Letear Racar}

ISSN: 2317-2347 - v. 10, n. Spécial (2021)

Todo o conteúdo da RLR está licenciado sob Creative Commons Atribuição 4.0 Internacional

\subsection{J'ai grandi, professionnellement et en tant que personnalité, avec la FIPF - par Doina Spita}

A l'occasion du 50ème anniversaire de la FIPF, la présidente de la Fédération brésilienne des professeurs de français, notre chère Denise Damasco, nous a convié.e.s à égrener quelques souvenirs. Je le fais avec grande émotion et une profonde reconnaissance, puisque, tel que je le déclarais lors de ma candidature pour un premier mandat à la vice-présidence de notre fédération, j'ai le sentiment indubitable d'avoir grandi, professionnellement et en tant que personnalité, avec la FIPF. En y réfléchissant, je vais en retenir trois leçons qui ont marqué mon existence.

J'ai connu la vie associative en 1990, dans un état d'esprit que les pays de l'Europe centrale et orientale comprennent certainement mieux que d'autres. Véritable mosaïque d'aires culturelles et linguistiques, ce vaste territoire a un dénominateur commun d'ordre historique : c'est l'expérience du régime totalitaire, une expérience d'environ un demi-siècle, lourde de conséquences au niveau sociétal et qui a entrainé, une fois que ces pays se sont lancés à l'apprentissage de la démocratie, des enjeux associatifs particuliers.

II m'arrive souvent d'évoquer à ce propos une phrase que j'ai entendu prononcer par Boris Cyrulnik. Je vais la citer ici, de mémoire : Pour donner sens à ce que l'on est, à ce qui nous entoure, il faut avoir une histoire et un rêve. C'est vrai pour les individus, c'est vrai aussi pour les entités collectives.

En 1990 donc, dans un mouvement d'émancipation qui a traversé toute la région, un groupe d'enthousiastes signait l'acte de naissance de l'Association roumaine des professeurs de français, ARPF, devenue plus tard, selon sa dénomination d'aujourd'hui, l'Association roumaine des professeurs francophones. Marqués par la sortie du communiste, ils étaient enthousiastes et aussi rêveurs, puisqu'ils rêvaient d'une Roumanie qui se sauve de l'isolement, ouverte et collaborative, une Roumanie dans laquelle l'enseignement du français soit un vecteur de la liberté. La constitution de l'ARPF fut ainsi un des premiers exercices de formation à la démocratie dans sa dimension participative. Elle s'inscrivait parmi les actes fondateurs de la société civile, accompagnés d'une prise de conscience de valeurs nouvelles, entre autres, celle de la responsabilité professionnelle collective. « II ne peut pas y avoir de positions d'attente, de passivité ! » - réclamaient, à juste raison, nos collègues. Le premier

projet fédérateur de la région en témoigne : La rénovation curriculaire en Europe Centrale et Orientale 


\section{Reuista Letras Racar}

ISSN: 2317-2347 - v. 10, n. Spécial (2021)

Todo o conteúdo da RLR está licenciado sob Creative Commons Atribuição 4.0 Internacional

: expertise et perspectives, déroulé entre 1995-1999, faisait découvrir aux associations leur rôle de conseil auprès des instances décisionnelles de l'éducation et, en l'assumant, de vecteur d'innovation. "C'est pour faire entendre ma voix que j'ai adhéré à l'association », déclarait une enseignante, comme tant d'autres, avec l'espoir d'une légitimité collective renforcée, garant du droit à veiller à ce qu'on garde le bon chemin. Effectivement, la plupart de nos associations sont loin de se contenter d'une attitude attentiste et les souhaits qu'elles expriment ne sont pas de simples utopies. Ce sont souvent au contraire autant de propositions qui peuvent inspirer, à tous les niveaux, des politiques linguistiques volontaristes, des stratégies et des projets. La légitimité de la FIPF, c'est la légitimité d'une voix planétaire. Ce fut une première leçon dont j'ai voulu vous faire part.

La deuxième leçon, je l'ai tirée de l'enquête entreprise lors de la préparation du Livre blanc de l'enseignement du français, dans les années 2013-2014. Elle s'appelle : penser l'avenir. L'enquête nous avait permis d'esquisser une sorte de portrait-robot de l'enseignant de français. Un personnage, si j'ose dire, à double visage. D'une part, il n'arrête pas de clamer son amour pour la langue française et la civilisation française et francophone, d'autre part, il se sent profondément « en souffrance » : découragé de la baisse constante de l'intérêt pour le français, abandonné au gré d'un chef d'établissement asservi aux lois du marché, fatigué des lenteurs institutionnelles face aux changement. II arrive que ce professeur, partagé entre les belles exigences de son métier et l'amertume devant l'opacité de bon nombre d'interlocuteurs et la désaffection croissante de la part du public, choisisse la vie associative, à l'intérieur de laquelle il espère retrouver son sourire et son optimisme. Peut-on le décevoir?

Il est de plus en plus évident que le français ne peut progresser sur le marché des langues que s'il est perçu comme un facteur de promotion sociale. Loin des voix moroses déplorant la baisse de son enseignement, nous devons affirmer la volonté de développer ensemble un savoir-faire en faveur d'approches méthodologiques rénovées, mieux ancrées dans ce que c'est le monde d'aujourd'hui et de demain. L'époque où l'on tirait profit de la seule beauté de la langue française est révolue. Ce que nous devons démontrer c'est que ce français, destiné à exprimer les détours imprévus de l'esprit, l'instantané des sensations, la petite musique enfouie dans le cœur de chaque homme, eh bien, que ce français est à même de garder son attrait. Qu'à cette demande correspond une offre de 


\section{Reuista Letras Racar}

ISSN: 2317-2347 - v. 10, n. Spécial (2021)

Todo o conteúdo da RLR está licenciado sob Creative Commons Atribuição 4.0 Internacional

qualité à la hauteur de l'attente qu'il suscite et que cette offre est capable d'entretenir son attrait et l'intérêt du public.

Les refrains moroses et parfois désespérés tels « le français perd du terrain face à d'autres langues », surtout à l'anglais, suggèrent un faux problème. II ne s'agit pas de "priorité ». Si l'on parle de plus en plus de " politique volontariste », il faut bien comprendre qu'il ne s'agit pas de la volonté envieuse de s'emparer de la position occupée par l'autre, mais de la volonté ambitieuse d'occuper une place à côté des autres et de militer en faveur du plurilinguisme propre à une culture démocratique. Notre Fédération doit avoir sa part, forte, généreuse et dynamique, dans l'élaboration de ce qui fait l'homme d'aujourd'hui et de demain. Penser l'avenir c'est faire preuve de souplesse, développer la capacité de s'adapter à tous les impératifs qui nous interpellent de la part de la société, enfin, être à l'écoute des jeunes. Le faire pour assurer la relève générationnelle, ce qui représente le défi d'un double déficit, aussi bien d'apprenants que d'enseignants de français. Penser l'avenir c'est innover, oser et agir.

La troisième leçon s'appelle solidarité. Elle n'est pas de date récente, mais c'est vrai qu'elle occupe le devant de la scène depuis que le combat mondial contre la pandémie nous oblige à serrer de plus en plus les coudes. L'origine de cette dernière expression née au XXe siècle est incertaine, mais une hypothèse veut qu'elle fasse référence au geste que font les manifestants d'entrecroiser leur bras les uns aux autres pour faire face à l'adversité. L'image est symbolique, puisque, paradoxalement, c'est l'à distance" qui nous a rapprochés, tel que l'a unanimement témoigné le groupe de collègues argentins participants à un atelier de formation à la vie associative que j'ai animé, en ligne, il y a moins d'un mois... Solitaires mais solidaires, pour reprendre Camus. Puisque nous ne gagnerons jamais seuls.

II y a plusieurs façons de décliner la solidarité associative : c'est en même temps le travail collégial, les projets collaboratifs ; les partenariats saints, construits sur le principe du donnant-donnant, le soutien contre la précarité aggravée par l'enseignement à distance ... et c'est aussi trouver ensemble, à l'heure des grands rendez-vous décisifs pour l'avenir de la planète, les bonnes réponses aux grands défis. Les constats et les analyses ainsi que les exemples de bonnes pratiques concourent à l'émergence d'un certain nombre de consensus, correspondant à des réalités largement partagées et à une volonté de rapprochement. Ces consensus issus du débat, de l'échange et du partage 


\section{Reuista Letras Racar}

ISSN: 2317-2347 - v. 10, n. Spécial (2021)

Todo o conteúdo da RLR está licenciado sob Creative Commons Atribuição 4.0 Internacional

contribuent au développement d'une culture associative qu'on va appeler ethos, une notion de plus en plus présente non seulement dans les sciences du langage mais encore dans les sciences humaines et sociales, voire dans les humanités. Un articulateur, une action commune, telle la volonté de grandir et de tenir bon, même (ou en particulier) quand ça ne va pas, et qui représente la marque d'un état d'esprit de développement. C'est aussi la vocation de notre fédération ! Nous en sommes tous témoins. Et peut-être aussi acteurs.

\subsection{La FIPF et les professeurs de français, «éclaireurs » de la Francophonie, par Dario Pagel}

Depuis sa création en 1969, la Fédération Internationale des Professeurs de Français (FIPF) sert et servira toujours la cause de l'essor et de l'épanouissement du français dans les différentes régions du monde, ce qui est sa tâche prioritaire, justifiant ainsi, par exemple, les différentes actions qu'elle mène jusqu'aujourd'hui avec ses partenaires nationaux, internationaux.

C'est comme sur un chemin sombre où il faut qu'un « éclaireur » ouvre la voie... Tel est le rôle de la Fédération internationale des professeurs de français qui, à travers ses quelques 80000 enseignants représentés sur les cinq continents, essaie de créer des espaces francophones partout où cela est possible. En effet, un cours de français en pays Massaï, chez des Indiens d'Amazonie ou chez des courtiers de Wall Street est un espace francophone qui se crée. Dans les coins les plus reculés du monde, l'enseignant de français demeure parfois la seule bougie qui éclaire la francophonie. Quand elle s'éteint, la francophonie s'éteint aussi. Lorsqu'elle résiste à un environnement qui ne lui est pas forcément favorable, survient son effet démultiplicateur ; un professeur dans une classe de cinquante élèves pendant trente ans de carrière : faites vous-même le calcul !

Cette francophonie, nous, enseignants de français, la défendons avec la force de notre énergie militante, en « mousquetaires ». Nous la voulons également moderne et concrète, c'est-à-dire adaptée aux réalités des femmes et des hommes de tous les continents, de toutes les races ou ethnies, de toutes les couches sociales.

Pour la valorisation et la vie associative de la francophonie, les professeurs de français représentent un capital inestimable d'expérience, irremplaçable de présence, d'influence à long terme et même, disons-le, de résistance. Regroupés dans des associations, toutes membres de la Fédération 


\section{Reuista Letras Racar}

ISSN: 2317-2347 - v. 10, n. Spécial (2021)

Todo o conteúdo da RLR está licenciado sob Creative Commons Atribuição 4.0 Internacional

internationale des professeurs de français, ces enseignants sont des forces vives et passionnées dans la construction d'une francophonie basée sur la solidarité, la diversité, l'amitié... Cette organisation associative des professeurs de français est remarquable et unique au monde qui va des régions internes des différents pays aux régions continentales, en concertation avec une fédération internationale.

Notre francophonie répond à l'idéal de Sédar Senghor, à ce que ce grand poète de la négritude qui est également l'un des fondateurs de la Francophonie institutionnelle a appelé le « rendez-vous du donner et du recevoir ", une espèce de plate-forme d'échanges où chacun déposera les meilleurs éléments de sa culture et emportera pour s'en imprégner les meilleurs éléments de la culture de « l'étranger ».

La FIPF et la FBPF sont conscientes de la responsabilité qui est accordée aux associations de professeurs de français, mais chaque professeur de français est lui aussi conscient que son association constitue un puissant noyau d'expression pour réagir contre la marche vers l'uniformité, en se mobilisant pour l'enseignement diversifié des langues qui sont la richesse du monde.

Les deux fédérations apportent à chaque moment une visibilité et une crédibilité à l'enseignement du français et aux actions de terrain. Le succès des congrès mondiaux de la FIPF et nationaux de la FBPF ont gagné le pari qu'elles avaient pris au moment de leur création, de ne pas défendre la langue française en se repliant sur le pré carré des pays où elle est la langue maternelle des apprenants, mais au contraire de rassembler aussi tous ceux qui l'enseignent comme langue seconde ou comme langue étrangère au service d'une francophonie ouverte.

Chaque enseignant a toujours trouvé dans les débats de ces grands rassemblements mondiaux et nationaux qu'il sera toujours essentiel de doter les professeurs de français de compétences fortes en langue française, en culture et en didactique, pour que chacun d'entre eux puisse devenir un opérateur confiant, dynamique, et omniprésent d'une francophonie ouverte répondant aux valeurs humaines et de paix, tout en affirmant ainsi le vrai rôle de l'enseignant qui est celui de former les jeunes et donc, de préparer l'avenir.

Les déclinaisons thématiques des congrès mondiaux, régionaux et nationaux permettent aux participants de porter leur réflexion sur les points forts de l'enseignement du français, mais aussi sur ses points faibles. Nous observons que dans certaines régions le français jouit d'un statut favorable 


\section{Reuista Letras Racar}

ISSN: 2317-2347 - v. 10, n. Spécial (2021)

Todo o conteúdo da RLR está licenciado sob Creative Commons Atribuição 4.0 Internacional

dans le système scolaire. Pourtant, dans d'autres systèmes éducatifs nationaux, il est menacé de disparaître, en raison de réformes planifiées par les gouvernements respectifs.

Les enseignants de français y ont été massivement conviés à donner leur vision d'un enseignement moderne de cette langue qu'ils défendent au quotidien, vision qui puisse redresser la courbe de la demande de cette langue dont certains n'hésitent pas à annoncer le déclin. Pour ce qui me concerne, j'ai depuis mon élection à la tête de la FIPF en 2000 une vision qui est restée constante. La visibilité du français dans le monde passe principalement par deux axes stratégiques importants : d'une part la formation et la fidélisation des enseignants ; et d'autre part l'enseignement du français dans les écoles des systèmes éducatifs des différents pays.

Aujourd'hui, l'avenir du français est, comme pour la plupart des langues, à la massification de l'apprentissage. II faut donc pour cela absolument éviter d'installer le français dans son confort de langue d'une élite sociale et culturelle relativement aisée. Plusieurs publications ainsi que différents travaux de congrès et colloques des Fédérations internationale et brésilienne des professeurs de français ont déjà débattu de la question de la présence du français, aujourd'hui précaire, dans les écoles de nombreux pays, principalement ceux dans lesquels le français est langue étrangère.

Le décloisonnement académique de l'étude de la langue et la vulgarisation des nouvelles technologies contribuent certainement à créer une image moins élitiste de l'apprentissage du français ; les divers cours de formation, les recherches universitaires, la poussée du français sur objectifs spécifiques, axé sur les besoins professionnels, adopté dans beaucoup d'institutions éducatives, dans certains pays latino-américains, permettent aux apprenants de trouver une raison « utile » à l'étude de la langue et leur ouvre des débouchés intéressants dans le monde du travail.

À présent, tous les acteurs du français langue internationale doivent interagir entre eux, soutenus par la France, le Canada, le Québec, la Belgique et les autres pays et gouvernements francophones, pour développer une politique cohérente et planifiée de la présence de la langue française dans les systèmes éducatifs d'une majorité de pays du monde. Et surtout, ils ne doivent pas oublier ce qui apparaît comme une évidence pour les acteurs de terrain que nous, enseignants de français, représentons modestement : pour la réussite de tout projet de politique de la langue française, il faudra s'appuyer sur les professeurs de français qui ont un regard éclairé sur la réalité de l'enseignement de cette langue dans leurs régions respectives. Structurés en associations et en réseau 


\section{Reuista Letras Racar}

ISSN: 2317-2347 - v. 10, n. Spécial (2021)

Todo o conteúdo da RLR está licenciado sob Creative Commons Atribuição 4.0 Internacional

mondial par la Fédération internationale et la Fédération nationale, ils doivent être les premiers interlocuteurs en matière de politique, de promotion et de diffusion du français.

Je souhaiterais que les réflexions du XXIlème Congrès brésilien des Professeurs de français donnent un dynamisme encore plus grand à notre vie associative qui, structurée, dévouée à la valorisation du français et composée de collègues nationaux, constitue un réseau ancré dans la réalité locale. Seuls les professeurs de français locaux connaissent le contexte et la culture éducative locale. Les associations sont donc des relais indispensables. Bien comprises, avec tact et confiance, avec aussi un soutien matériel et avec la reconnaissance qu'on leur doit, elles pourraient constituer un réseau de diffusion extraordinaire pour que la langue française permette de consolider davantage un dialogue fait de respect mutuel et d'amitié, dans la nécessaire diversité linguistique et culturelle de ce continent européen. Voilà la langue qui fait la différence.

\subsection{La FIPF et le regard d'une enseignante membre à une association adhérente à la FIPF, par} Denise Damasco

À Salvador, en 1998, c'était la première fois que j'ai compris que l'Association des Professeurs de Français du District Fédéral appartenait à un réseau plus élargi. D'ailleurs, ce mot « réseau » ne faisait pas de sens pour moi à cette époque-là...C'était la Xlllème édition du Congrès brésilien des professeurs de français et si je me souviens bien, la réélection de Dario Pagel comme président de la FBPF'1. De là, à ma réalité d'enseignante de français dans le cadre de l'Alliance française de Brasilia et affectée au système public du District Fédéral, il y avait un long écart. En tout cas, après la séance d'ouverture de ce congrès, dans une belle salle d'un hôtel de cette ville, j'ai vu M. Pagel s'approcher pour nous saluer, j"étais avec la coordinatrice de l'Alliance française de Brasilia, Mme Saad, qui m'a fait connaître M. Pagel et la FBPF.

En 2014, lors de l'organisation de la 6ème Rencontre Nationale des Présidents des Associations des Professeurs de Français à Brasilia, en tant que présidente de l'APFDF, j'ai pu saisir cet effet de cascade et ce que réseau voulait dire, lorsque nous sommes dans le monde associatif. Être en réseau

\footnotetext{
1 Selon le site de la FBPF <http://fbpf.org.br/site/qui-sommes-nous/>
} 


\section{Reuista Letras Racar}

ISSN: 2317-2347 - v. 10, n. Spécial (2021)

Todo o conteúdo da RLR está licenciado sob Creative Commons Atribuição 4.0 Internacional

pour moi comprend s'entraider de façon à faire rayonner nos objectifs communs. Quand nous sommes en réseau, nous sommes capables de surmonter nos difficultés de façon assez légère et soudée.

Défi lancé en 2017 pour moi, organiser un bureau pour faire renaître la FBPF qui voyait son organisation juridique s'écouler d'une année à l'autre, j'ai pu comprendre que cette tâche allait s'accomplir avec l'appui de la Fédération internationale des professeurs de français. C'est à l'occasion du Congrès brésilien des professeurs de français à Aracaju que j'ai pu faire connaissance de Mme Doina Spita, qui venait nous joindre au Brésil et qui nous parlait des enjeux internationaux du réseau fédératif. Là, de mon regard associatif local, j'ai compris que la force du réseau nous soutient et nous mène à penser à des projets plus ouverts et plus diversifiés.

Alors, c'est avec beaucoup de simplicité que j'ai pu fixer un rendez-vous avec M. Stéphane Grivelet, secrétaire général de la FIPF, 8 rue Beauvais, à Paris en 2018. Entourée des collègues si gentils, telle que Mme Isabelle Desnouailles et de M. Diego Fonseca, notre collègue brésilien à la FIPF, que j'ai eu l'occasion de rapporter les actions que le bureau de la FBPF entamait pour la reprise juridique et fiscale de la FBPF. La vie fédérative au Brésil existait en dépit de ce manque d'ordre juridique. Avec beaucoup de respect nous avons pu reprendre la situation financière de la FBPF et faire la mise à jour de nos cotisations auprès de la FIPF. De cette conversation, pendant un matin d'automne, j'ai compris que la FBPF pouvait vraiment faire face à ses défis : nous n'étions pas seul.es.

Pour une présidente de fédération novice, le plus important pour ne pas se perdre, c'est analyser le terrain pour voir sur qui nous pouvons compter. Avec la FIFP, j'ai compris que je marchais sur un sentier battu. De notre côté, les promenades sur des sables mouvants étaient également finies. Quand j'ai proposé à la FIPF une gestion financière tripartite de la 22ème édition du congrès brésilien des professeurs de français à Brasilia, la réponse que j'ai eu c'était : oui, nous allons vous aider. L'appui institutionnel de la FIPF à la FBPF venait de nous rendre plus sûres de nos actions. La présidente de I'APFDF à l'époque, Mme Rosana Correia et moi, en tant que présidente de la FBPF, nous sommes parties pour l'organisation de notre congrès, ayant la FIPF. Reconnaissant que la FIFF mène une aide formelle aux congrès régionaux et pas aux congrès nationaux, nous avons pu avoir Mme Doina Spita chez nous et cette aide pour le congrès de Brasilia a été fondamentale pour nous faire mieux comprendre, ce que réseau exprime. Être en réseau nous induit à avoir de projets plus ambitieux et ne pas nous arrêter face à des difficultés locales. Un réseau national et international nous soutien. 


\section{Reuista Letear Racar}

ISSN: 2317-2347 - v. 10, n. Spécial (2021)

Todo o conteúdo da RLR está licenciado sob Creative Commons Atribuição 4.0 Internacional

Après avoir célébré le cinquantenaire de la FIPF à Brasilia lors de notre congrès national, nous avons décidé de rêver ensemble à ce français qui est « solaire », une langue qui peut atteindre à toutes et à tous. Entre 2020 et 2021, nous avons préparé notre candidature pour devenir le pays-siège de la 16ème édition du congrès mondial des professeurs de français. Cette fois-ci, notre candidature n'a pas été retenue, ce qui nous a fait comprendre que nous devons encore mieux nous préparer. Mais un détail est important, nos liens sont vraiment soudés, ces liens tissés à travers des projets communs, ces liens qui nous rapprochent et qui nous font avancer dans les années à venir, avancer en tant que fédération brésilienne et la fédération internationale des professeurs de français.

\subsection{La vie associative et les occasions de formation aux nouveaux débouchés en FLE : les actions de la FBPF et l'APFDF, par Waldemar Oliveira, président de l'APFDF}

La vie associative se fait remarquer même à notre insu. Un jour on est dans une salle et nous écoutons un ancien président Dario Pagel rapporter son histoire auprès de la FIPF et aussi la voix de sa vice-présidente...tout cela était modéré par la présidente de la FBPF...un autre jour, on est là comme représentant d'une association, nous aussi.

Tel est le constat que l'on a pu rapporter dans le cadre de la XXle Semaine Universitaire de l'Université de Brasilia, où l'on a dressé un bilan des actions de l'Association des professeurs de français du District fédéral (APFDF), à partir de nos impressions et aussi de quelques lectures. Pour mener à bien cette intention, nous avons présenté l'historique de nos actions et aussi le reflet de nos initiatives, dont l'impact se ressent même chez ceux ignorant l'existence d'une vie associative en dépit de s'en bénéficier directement. Ce constat a abouti à une réflexion sur le fait d'animer une vie associative, notamment face à un contexte où la pratique pédagogique s'avère des plus solitaires même si l'on s'insère dans une filière formée de nos homologues avec une formation initiale commune.

Actions en réseau, bénévolat et l'envie de regrouper des efforts au nom de la diffusion de l'enseignement du français au Brésil, c'est le constat qu'on fait, lorsqu'on entre en contact avec la vie associative, plus précisément, dans la sphère de la langue française, ces éléments voient le jour. En acquiescement à ces premières impressions, Cuq (2016) affirme : 


\section{Reuista Letear Racar}

ISSN: 2317-2347 - v. 10, n. Spécial (2021)

Todo o conteúdo da RLR está licenciado sob Creative Commons Atribuição 4.0 Internacional

Les associations ne sont pas des organisations politiques, ni des syndicats, mais des lieux collectifs d'organisation de la vie professionnelle et de partage de valeurs communes. Or, si la vie professionnelle des professeurs de français est bien sûr largement la même que celle de beaucoup d'autres professeurs, elle est aussi très singulière. (CUQ, 2016, p. 7)

Bien au-delà du simple exercice de fonction, participer à la vie associative entraîne vivre ensemble. Face à ce scénario, le réseau se trame, les contacts s'entament. Chaque enseignant, chaque étudiant s'inscrit dans une association locale et y partage ses centres d'intérêt. Cette association, à tour de rôle, est membre d'une fédération, avec ses autres confrères d'un même pays ou sous-continent. Ces liens se tissent jusqu'au niveau global, sans que l'on se passe de l'objectif premier, le même qui a fait tout enseignant, tout jeune étudiant adhérer à la vie associative. Quel que soit le niveau, l'engagement est le même ; les proportions gardées, chacun s'apprête à jouer son rôle tant à la promotion de la langue française qu'à la mise en œuvre des ressources permettant de perfectionner leurs pratiques.

Cependant, le travail sur réseau est peu faisable si l'on ne prend pas en compte le bénévolat de ceux qui doivent le gérer. À présent, les membres du bureau exercent leurs fonctions sans avoir droit à aucune rémunération, que l'on soit au niveau local (APFDF) ou au niveau national. En effet, on finit par boucler, dans notre tram-tram, nos tâches associatives parmi nos autres - et pas moins nombreuses - contraintes journalières. Une abnégation qui donne des résultats : cette envie du partage, cette motivation quasiment franciscaine de donner et(pour) recevoir renforcent l'élan premier de la vie associative lorsque l'on y anime la formation continue, non seulement au nom du sens de l'appartenance mais dans un scénario où une toute petite étincelle représente un phare intense, vis-àvis d'une mer où l'on s'y met sans perspective de retrouver un bon port.

Tels sont les facteurs qui relèvent l'importance de la réactivation de la Fédération brésilienne des professeurs de français, en 2017. La formation continue se voit comme capital à la constitution du savoir-être et du savoir-faire de l'enseignant, comme l'évoquent Damasco, Moreira et Magalhães (2020). Du coup, des actions entreprises ponctuellement, souvent dépendantes des projets et des prises en charges personnelles, ont pris de l'ampleur nationale à l'aide des subventions des partenaires premiers de la langue française. Grâce à l'intervention des Services de coopération et d'action culturelle, ainsi que leurs analogues, l'accord gagnant-gagnant s'établit, ce qui corrobore l'expérience 


\section{Reuista Letras Racar}

ISSN: 2317-2347 - v. 10, n. Spécial (2021)

Todo o conteúdo da RLR está licenciado sob Creative Commons Atribuição 4.0 Internacional

précédente de la Fédération à l'organisation des appels à participation à des différents stages de formation continue. Le membre d'une association locale est subventionné, ce qui fait étayer son savoirfaire pédagogique. L'association, à son tour, gagne un formateur local capable de mutualiser les connaissances qu'il compte être essentielles à retenir. Au niveau national, la Fédération a aussi son rôle à jouer : animer la vie associative par l'intermédiaire de l'amélioration des conditions de l'enseignement et des recherches de langue étrangère. Sitôt on met un terme à ce cycle, sitôt on le reprend.

Comme l'on doit toujours être reconnaissant, l'actuel bureau de l'APFDF a une histoire qui le précède. Le bureau actuel a un délai, certes ; l'envie de faire tourner la girouette de la vie associative n'est jamais exaucée dans la mesure où elle est notre instrument de lutte, notre occasion à la fois de foisonner la formation continue et de chercher de nouvelles occasions quidam le souhaitant. Avec moi, au bureau, il y a aussi : Josely Soncella, vice-présidente ; Katia Silva et Denilson Alves (première et deuxièmes secrétaires, respectivement) ; Denise Damasco et Paulina González (première et deuxième trésorières, responsables de contrôler à la loupe nos finances et que je remercie énormément). Dans notre bureau, on emprunte la devise Unité dans la diversité, vu qu'on connaît tous un parcours bien particulier, et dans nos pratiques, et dans notre formation.

À propos de notre APFDF, cette association a vu le jour le 8 mai 1968, quand 26 enseignants de français ont mis au monde cette association qui visait rassembler les enseignants, échanger des idées de façon efficace pour diffuser et perfectionner l'enseignement de la langue et de la civilisation françaises dans le système éducatif. Qui dit 68, d'ailleurs, dit Edson Luís, dit Passeata dos Cem Mil, crie à tue-tête « il est interdit d'interdire »; cette année ballotée entre l'ombre de la répression accrue et la lumière venue d'un nouveau monde semble elle aussi dicter notre vocation en tant qu'association. Entre l'envie de se rassembler et des périodes d'inactivité, nous sommes arrivés au jubilé en juin 2018, avec un seul désir : rendre lumière là où l'on insiste de sombrer, soit pour cécité, soit pour inertie.

Ainsi on emprunte le sentier. Lorsque l'enseignement du français s'est vu marquer le pas, celui-ci s'est fait pallier grâce à l'intervention de la vie associative. Même aujourd'hui, quand on pense aux journées de confinement plus serrées comme un lointain souvenir, ces petits écrans se poursuivent en tant que notre salle de classe, chaque nouvel onglet comme une fenêtre ouverte sur la rue. La vie associative en est allée de même, en repérant les initiatives de nos associés, dont certaines d'entre 


\section{Remita Letear Racar}

ISSN: 2317-2347 - v. 10, n. Spécial (2021)

Todo o conteúdo da RLR está licenciado sob Creative Commons Atribuição 4.0 Internacional

elles viennent d'être à votre portée, faisant que d'autres s'y inspirent. D'où la mise en œuvre d'un livre racontant nos mémoires, on a poursuivi le programme de formation continue, on a rétabli nos contacts entre vie universitaire et vie associative, réputés essentiels au savoir-faire enseignant.

Plus précisément, une initiative se fait remarquer. Le livre Génération de profs : histoire, mémoires, actions a vu le jour dans le but de matérialiser son rôle : préserver notre mémoire, raconter notre histoire, revisiter notre album de mémoire, en quête de pérenniser nos actions depuis la dernière réactivation. Deux citations de ce livre soulignent un sens de l'appartenance présent dans toute vie associative :

Nous pouvons sans doute affirmer que ces frontières se retrouvent dans le passage de la vie d'étudiant. e de français à un parcours d'enseignant.e de la langue française. D'après le célèbre sociologue Mannheim, les générations ne sont pas remplacées par d'autres. Elles se succèdent. [...] Par conséquent, la génération représente une succession de générations étant donné que la seule idée de contemporanéité des sujets ne suffit pas à créer une génération. (Témoignage de Denise Damasco - $s / p$ - en vue de publication)

Aujourd'hui je suis professeure de français et je peux dire que je vis mon rêve ! Et c'est grâce à André que j'ai pu le faire. Dans les mots de William Arthur Ward "Le professeur médiocre raconte. Le bon professeur explique. Le meilleur professeur démontre. Le grand professeur inspire." Je tiens à témoigner ici que mon professeur André Santiago, lui, il inspire ses apprenants ! Merci infiniment, mon cher professeur et ami ! (Témoignage de l'enseignante Erica Cirino - s/p)

Autre action est le programme de formation continue de l'APFDF, qui a gagné un nom Formations en route - et a assumé un nouveau format - en ligne. À l'intention d'organiser les séances de rétroaction de nos membres, devenus formateurs par l'intermédiaire des appels à participation propres et aussi organisés par nos partenaires, cet espace privilégié de rencontre a permis l'échange d'expériences de plus de 200 professeurs inscrits, sur des sujets pertinents à notre savoir-faire pédagogique. Telles pratiques suscitent naturellement la réflexion sur son propre parcours professionnel. L'appropriation de nouveaux outils, la confirmation de ses nouvelles pratiques, tous deux facilités par ces échanges, facilitent la création de nouvelles stratégies qui resignifient ces pratiques pédagogiques, améliorant la prise de conscience du contact avec un savoir-faire pédagogique constitué autrement. 


\section{Reuista Letras Racar}

ISSN: 2317-2347 - v. 10, n. Spécial (2021)

Todo o conteúdo da RLR está licenciado sob Creative Commons Atribuição 4.0 Internacional

L'avènement du SEMIFRA est aussi un autre exemple. Le Séminaire régional de recherches d'expression française a vu le jour dans le but d'être un espace de discussions sur la recherche et la formation des professionnels qui développent des études concernant l'enseignement et l'apprentissage du français. Au cours des trois premières éditions, cet espace privilégié a accueilli des professeurs, des conférenciers et des étudiants, dans les domaines de littérature ou de didactique de l'enseignement des langues. À ce stade, comprendre le contexte global y joue également un rôle important, avec des activités de formation continue, menant à la réflexion sur les chemins à suivre dans le processus de l'enseignement-apprentissage.

II n'allait pas de soi former à distance dans un domaine où le contact présentiel s'avère essentiel. Animer la formation continue, d'ailleurs, ne nous était pas quelque chose de nouveau. Depuis la réactivation de l'APFDF en 2013, nous avons mis sur notre règlement intérieur non seulement d'enrichir le répertoire de la pratique pédagogique de nos professeurs, mais aussi de promouvoir des séjours linguistiques en langue française. Ceci dit, avant le confinement, nous avons pu envoyer au moins deux de nos membres par an à des stages de courte durée offert par des écoles confirmées comme le CAVILAM et CLA, et aussi nous avons subventionné la participation à des congrès au Brésil et à l'étranger (XXI CBPF, FIPF Liège 2016, SEDIFRALE 2018). Le fait que notre Association se soit vu régulariser sa situation fiscale et notariale a rendu possible la réalisation, pour la première fois en Amérique du Sud, des universités BELC-2018 (programme de formation continue organisée par France Éducation International, organisme responsable de l'application des tests de connaissance de français DELF-DALF et aussi des échanges des professeurs, étudiants et pratiques pédagogiques). Auparavant, on a eu nos éprouvettes : la Semaine d'immersion, 2016, ainsi que les Journées de formation et d'immersion, en 2017 ont été entreprises, respectivement, à l'Ambassade de France et dans un hôtel, toujours en quête de dynamiser la vie associative par l'intermédiaire de la promotion de la formation de nos membres. Après La première expérience BELC, nous avons subventionné la participation de trois autres professeurs aux éditions suivantes de l'Université BELC - Buenos Aires, 2019 et à nouveau Brasilia, 2020. Si le nouveau SEMIFRA aura lieu virtuellement, les trois autres éditions précédant l'actuelle ont été faites en présentiel, permettant le partage d'expériences entre des chercheurs débutants et confirmés, en concomitance avec des professeurs qui y ont également trouvé 


\section{Reuista Letear Racar}

ISSN: 2317-2347 - v. 10, n. Spécial (2021)

Todo o conteúdo da RLR está licenciado sob Creative Commons Atribuição 4.0 Internacional

l'occasion de rapporter leurs expériences. En effet, les recherches sont toujours à faire même si l'on n'est pas en milieu universitaire.

Comme on peut l'observer, la liste d'opportunités est des plus importantes. Mais pour ces débouchés nous faisons à la fois comprendre la formation initiale et tâter les perspectives dans l'avenir en français, une question s'impose : la vie associative, à quoi bon s'y mettre ? De notre part, notre réponse est positive. Et j'oserai même d'ajouter : on ne peut pas avoir toute action de cette nature si on insiste d'agir tout seul. Plus précisément, les partenariats s'établissent lorsque deux personnes morales concertent leurs objectifs. Notre association a été réactivée, ses statuts ont été approuvés, les cotisations annuelles ont été recueillies, les situations fiscales, budgétaires et notariales ont été régularisées. À titre d'exemple, dix ans plus tard, Brasilia a accueilli un Congrès brésilien de professeurs de français. Un événement de telle ampleur ne serait pas possible si des fournisseurs et prestataires n'étaient pas payés ; des académiciens confirmes n'auraient jamais rejoint le comité scientifique ; des stands avec des partenaires n'auraient pas pu être installés. Bref, rien n'aurait été possible sans que notre situation fût mise en règle auparavant, et il en va de même quand on pense aux autres initiatives qui ont encouragé la formation continue des associés. En outre, la réactivation de la Fédération brésilienne des professeurs de français a permis aux membres des associations de se former en continuité, toutes modalités confondues. De ce fait, intégrer la vie associative est convenable nous rendant visibles, par l'intermédiaire du bénévolat d'innombrables personnes, dans la mesure où cette intégration prête la voix non seulement aux désireux de perfectionner leurs pratiques pédagogiques mais aussi aux avides de partager telles bonnes pratiques avec nous.

Autre facteur important à souligner demeure sur la clarté des appels à participation de ces formations. Qui que soit l'organisateur - l'APFDF et/ou ses partenaires - la définition des prérequis et de contraintes à l'aide des règlements rassure les candidats qui, une fois retenus, s'engagent à retransmettre, par l'intermédiaire des formations, les connaissances que celui-ci aura jugées plus pertinentes. Au niveau local, nous avons une disposition interdisant la participation des membres actuels du bureau à tout appel organisé par l'Association elle-même, ce qui évite à ce stade toute sorte de favoritisme.

\section{Conclusion}




\section{Reuista Letras Racar}

ISSN: 2317-2347 - v. 10, n. Spécial (2021)

Todo o conteúdo da RLR está licenciado sob Creative Commons Atribuição 4.0 Internacional

Pour conclure, les auteurs se permettent également de poser une question : la vie associative aurait-elle lieu, même chez les jeunes professeurs ou les débouchés ? En guise de trouver une réponse, il faut ôter la casquette de président et de mettre celle de quelqu'un qui a vécu trois moments distincts au cours de cette vie associative - inactive, « favoritiste » et active. Lorsque l'on n'a pas de vie associative, tout jeune professeur désireux de comparer le vrai monde à celui a été dépeint sur ses manuels ne s'attend pas à grand-chose. Lorsque la vie associative est repliée sur elle-même, plus précisément sur ses directeurs en compagnie de ses suppôts, la chance des débouchés est presque nulle. En revanche, lorsqu'on a une vraie vie associative, au moins une possibilité s'ouvre à ce jeune professeur - lui accordant l'exploration de la réalité, voire aux débouchés qui se sont apprivoisés par le métier, souvent inspirés par leurs futurs collègues : ces anciens enseignants à l'éducation de base.

Autre facteur utile à mentionner repose sur le fait que notre savoir-faire pédagogique est des plus solitaires, même si l'on est en état d'immersion linguistique. Cette solitude, cette monotonie propre des routines quotidiennes se brisent par le sens de l'appartenance. Ce savoir-faire pédagogique peut nous rassurer dans la mesure où l'on estime être nécessaire aller au-delà de la formation du premier cycle face au tram-tram des leçons parfois trop répétitives. Le besoin d'une formation constante, aussi bien que la possibilité de la faire, grâce au sens de l'appartenance, est le oui définitif aux nouveaux débouchés envisageant aussi le métier de formateurs, dans un cercle vertueux étayé des savoir-faire et savoirs-être pédagogiques.

Si nous avions un conseil ou une suggestion à faire aux associations et à leurs enseignants, nous leur demanderions de défendre une image moderne du français. En notre qualité de trois générations de militants de la langue française, nous avons coutume de rappeler que le français, qui est pour nous une langue d'adoption, doit effacer cette image de langue de l'élite pour devenir une langue accessible à tous. Nous pensons que ce n'est qu'à ce prix que nous réussirons la bataille du plurilinguisme, et développerons l'enseignement du français. 


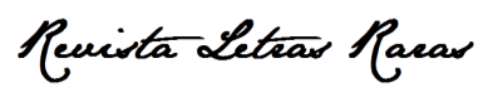

ISSN: 2317-2347 - v. 10, n. Spécial (2021)

Todo o conteúdo da RLR está licenciado sob Creative Commons Atribuição 4.0 Internacional

CRediT
Reconnaissances: Ce n'est pas applicable.
Financement: Ce n'est pas applicable.
Conflits d'intérêt: Les auteurs certifient qu'ils non pas d'intérêt commercial ou associatif sous un conflit d'intérêt
par rapport au manuscrit.
Approbation éthique: Ce n'est pas applicable.
Contribution des auteurs:
Denise Gisele de Britto Damasco - Conception de l'étude, Administration du projet, Validation, Visualisation,
Rédaction/préparation du manuscrit ('original), Rédaction du manuscrit - révision et édition.
Dario Pagel - Conception de l'étude, Administration du projet, Validation, Visualisation, Rédaction/préparation
du manuscrit ('original), Rédaction du manuscrit - révision et édition.
Doina Spita - Conception de l'étude, Administration du projet, Validation, Visualisation, Rédaction/préparation
du manuscrit ('original), Rédaction du manuscrit - révision et édition.
Waldemar Oliveira de Andrade Junior - Conception de l'étude, Administration du projet, Validation, Visualisation,
Rédaction/préparation du manuscrit (l'original), Rédaction du manuscrit - révision et édition.

\section{Références}

CUQ, J. P. Les associations de professeurs de français et la FIPF au service de la francophonie, In : Le français à l'université, n.1 et n. 2, 2016, p. 3.

, La vie associative des professeurs de français, un engagement entre intérêt et idéalisme, In : Synergies Brésil, nº 7 - 2009, pp. 154-159.

DAMASCO, D. G. B.; MOREIRA, H. C. A.; MAGALHÃES, P. A. A. La vie associative au Brésil : enjeux actuels pour la formation continue des enseignants de français et le travail associatif en réseau. In: CONGRES BRESILIEN DES PROFESSEURS DE FRANÇAIS, 22., 2019, Brasília, Actes du XXIlème Congrès Brésilien des Professeurs de Français. Édition spéciale de la Revue Letras Raras: Campina Grande. EDUFCG. nov. 2020, p. 464-473.

FRANCHON, C. Le mot de la présidente du comité scientifique. In: CONGRES BRESILIEN DES PROFESSEURS DE FRANÇAIS, 22., 2019, Brasília, Cahier de Résumés du XXllème Congrès Brésilien des Professeurs de Français (CBPF), 147-149. Disponivel em: <file:///C:/Users/denis/Downloads/2023-9641-1-PB\%20(3).pdf>. Acesso em out. 2021. 